\title{
Correlations between Hand Dexterity and Bimanual Coordination on the Activities of Daily Living in Older Adults with Mild Cognitive Impairment
}

\author{
Prathomchai Rattanawan \\ Department of Physical Therapy, School of Allied Health Sciences, Walailak University, Nakhon Si Thammarat, \\ Thailand
}

\section{Keywords \\ Hand dexterity - Bimanual coordination - Activities of daily living $\cdot$ Mild cognitive impairment}

\begin{abstract}
Background/Aims: Many motor impairments are present in older adults with cognitive decline. One of them is the impairment of hand dexterity and bimanual coordination that result in poor functional ability in the activities of daily living (ADL). This study investigated the effects of hand dexterity and bimanual coordination declination on the sub-domains of $\mathrm{ADL}$ in older adults with mild cognitive impairment (MCI). Methods: Thirty-one senior individuals with $\mathrm{MCl}$ were recruited in this study. The Purdue Pegboard Test was used to measure hand dexterity, and bimanual coordination was assessed by the continuous circle-drawing task. Their ADL were assessed with the General Activity Daily Living questionnaire. Results: The correlations analysis showed an association between the dominant hand and bimanual dexterity with the domestic domain of ADL and all conditions of hand dexterity with the complex domain of ADL. Moreover, the multiple regression analysis showed that the predictor of the greatest effect for domestic and complex domains was dominant hand dexterity. Discussion/Conclusion:
\end{abstract}

These results revealed that dominant hand dexterity strongly affected domestic and complex ADL in older adults with $\mathrm{MCl}$. There were age-related changes regarding lateral asymmetrical motor reduction, especially in cognitive tasks. However, complex tasks involving cognitive function may need dominant, nondominant and bimanual hand dexterity.

(c) 2022 The Author(s).

Published by S. Karger AG, Basel

\section{Introduction}

Cognitive impairment is a major clinical and public health problem that involves the impairment of episodic memory, languages, and problem-solving skills. More importantly, it affects the quality of life in older adults and their families [1]. Thus, it is important to identify the factors that may be affected after cognitive impairment.

Patients with Alzheimer's disease (AD) exhibit a progressive impairment of episodic memory and other cognitive capability, which affects their functional abilities [2]. However, in the early stages of $\mathrm{AD}$, also known as mild cognitive impairment (MCI), they show a slightly impaired cognitive capability and functional abilities [3]. Many motor impairments may emerge as a phenotype of cognitive

Correspondence to: 
decline [4]. A mild decline in fine hand motor function and complex hand motor function were found in older adults and affected to less able to control or adjust the amount force to fit the task, which is essential for activities of daily living (ADL) such as pouring milk, removing money from a wallet, and writing. Especially, hand dexterity is an important component to completely perform the tasks in ADL and the hand dexterity showed a decrease in older adults with MCI [5-9]. Changes in brain from neurodegenerative dementia (e.g., brain atrophy, neuronal loss, or synaptic dysfunction) lead to difficulty to learn movement causing both cognitive and motor dysfunction in MCI [10]. A deficit in the dominant hand, nondominant hand, or bimanual dexterity is associated with functional loss, particularly affecting the deficit of instrumental ADL (IADL) [11]. IADL require more complex neurophysiological processing capacity than basic ADL (BADL) and therefore, are more prone to deterioration triggered by cognitive decline and seem to be most severely affected in elderly with MCI more than without MCI. The elderly with MCI requires more time and less accuracy while solving tasks such as planning a bus route, packing specific item in a picnic basket, or taking medication [12]. The nature of hand movement requires visual search [5], motor speed skill [6], as well as attention allocation and motor planning [13]. Therefore, the main important functional skills, which are to control the attention, working memory, planning, judgment, task flexibility, and inhibition [14], seem to be strongly influenced by the performance of hand functions as a result of the reflection on the skilled fingers in grasping, lifting, and manipulating objects. Many daily bimanual tasks require precise force adjustments for stabilization and manipulation as seen in the hand dexterity, grip strength, or bimanual coordination.

Moreover, a bimanual task presents a challenge for fine motor control and requires the precise spatiotemporal coordination of both hands. Age-related declinations in coordination depend on the type of tasks and bimanual performance [15-17]. Older adults exhibit a greater breakdown in temporal coordination and slower movement in execution time than younger adults, especially in conditions requiring bimanual asymmetrical coordination [16]. In general, bimanual functional skills depend on the intra and interhemispheric neural networks involving the primary motor cortex, supplementary motor area, premotor cortex, cingulate, and posterior parietal cortex [18]. These areas are necessary for controlling bimanual movement and the connection between two hemispheres via the corpus collosum [19]. Structural and functional changes in the aging brain include the corpus

Effects of Hand Performance Declination on ADL collosum that affects bimanual coordination. From magnetic resonance imaging studies, the appearance of widespread gray and white matter deteriorations have been shown in aging [20] and a pronounced progression in the frontal area [21]. The older adults who have exhibited cognitive decline demonstrate an even larger reduction of connectivity between the intra and interhemispheric neural networks [22-24]. A continuous bimanual circledrawing that relies on the coordination between hand functions has been investigated in previous studies [25, 26]. They have found that continuous circle-drawing was not different between young and older adults. However, there are few studies reported on the effects of changes in the intra and interhemispheric connections controlling bimanual coordination [26-28].

Current data on the brain control function in each and in bimanual hands for performing ADL in older adults with MCI is limited. Therefore, the aim of this study was to investigate whether age-related hand functional impairments as a result of the reduction of intra and interhemispheric neural connections have any impacts on the hand performance to perform sub-domain of ADL impairment. This study determined the effects of hand dexterity and bimanual coordination declination on the subdomain of ADL in older adults with MCI.

\section{Materials and Methods}

\section{Participants}

This study was carried out at a selected community in Nakhon Si Thammarat from March to August 2020. A total of 49 participants were initially recruited in this study, 18 participants were excluded from this study which consists of a participant showed sensation impairment, a participant showed anxiety and depression, and 16 participants had Montreal Cognitive Assessment (MoCA) score more than 26 or less than 17 (cutoff score was set at 26 had sensitivity of $90 \%$ to detect MCI [29]), as showed in Figure 1 . Total of 31 participants (11 males and 20 females) with different socioeconomic backgrounds gave consent to participate in the study. The inclusion criteria were (1) participants were in the agegroup of 60-79 years old; (2) participants had right-handed dominance; and (3) participants were well oriented to time, place, and persons. All participants gave written informed consent for their participation and for the assessment of their cognitive level by the MoCA, and the assessment of their anxiety and depression status via face-to-face interview. All information was clearly explained to the participants along with questions and answers with detailed information of the protocol prior to the study. The study protocol was proved by the Ethics Committee of Walailak University (Reference No. WUEC-20-069-01).

Hand Dexterity Assessment

Hand dexterity was assessed using the Perdue Pegboard Test (PPT: Lafayette Instrument Co., Model 30,020) and the standard- 


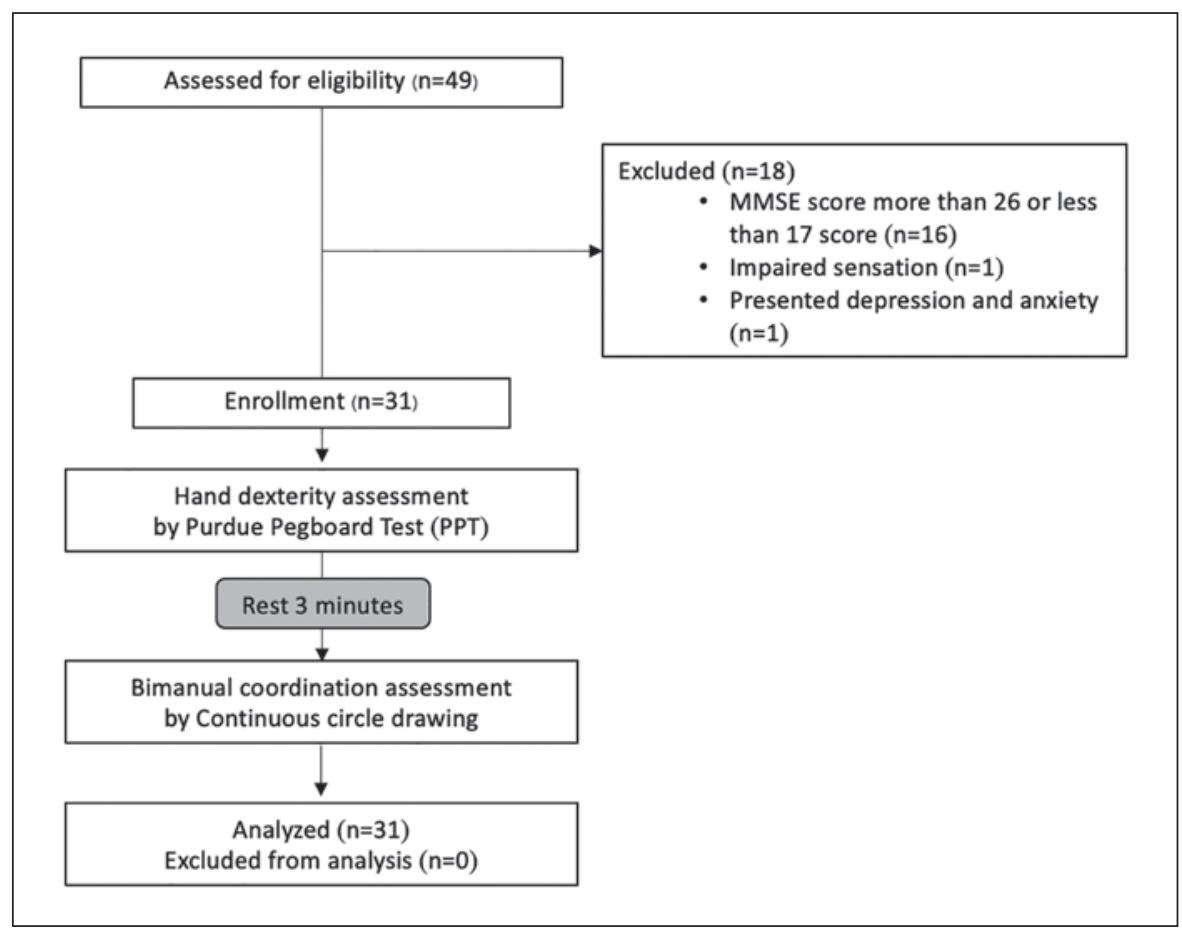

Fig. 1. Study flow diagram.

General Activity Daily Living Assessment

The standard questionnaire for General Activity Daily Living (GADL) was used to evaluate functional ADL for each participant by face-to-face interview. In short, the GADL shows a hierarchical structure with three components of more specific activities as demonstrated in the previous study [31]. In summary, the first component is BADL or self-care ADL, including changing clothes, using the toilet, bathing or showering, getting into/out of bed or a chair, and eating. The GADL covers the IADL; the first component of IADL is the domestic domain including the ability of doing household chores, telephone calling, meals preparation, and doing the laundry. The second component of IADL is the complex domain including the ability to manage financial matters, to shop, to take medication, and to go out alone on public transportation. Each domain showed good internal consistency $(>0.800)$ and domestic and complex's accuracy was higher for the distinction between MCI and AD. This assessment included an interview with the participants to investigate their ability to respond in each activity. The participants were also instructed to provide their accurate answers for each activity.

The scoring for the evaluation of each activity was divided into three scales: 2 means the participant is independent to perform the activities without help or supervision, 1 means the participant partially needs supervision, help, or special equipment to perform the activities, and 0 means the participant needs constant helps, supervision, or special equipment to perform the activities.

\section{Procedure}

The participants were screened for their cognitive level by the MoCA, including their anxiety and depression status. Since educational adjustment has been found to affect the MoCA scores [32], the level of cognitive education was categorized as $1-4,5-8$, 
Table 1. Baseline demographic characteristic of the study subjects

\begin{tabular}{ll}
\hline Characteristics & 31 participants, $n(\%)$ \\
\hline Gender & \\
$\quad$ Male & $11(35)$ \\
Female & $20(65)$ \\
Age-group, years & \\
$60-69$ & $17(55)$ \\
$70-79$ & $14(45)$ \\
Formal education, years & $12(39)$ \\
$1-4$ & $4(13)$ \\
$5-8$ & $7(22)$ \\
$9-12$ & $8(26)$ \\
$12+$ & $7(22)$ \\
Diseases, $n$ & $12(39)$ \\
Non & $12(39)$ \\
One disease & \\
Two or more diseases &
\end{tabular}

9-12, and more than 12 years. However, the education effects of 1 point were added for participants with 12 years of education on their MoCA score (if <30).

All 31 eligible participants sat comfortably on their chair and placed their arms on a table in a position ready to reach and pick up an object in front of them. The procedure was first explained and the assessment of PPT was demonstrated step by step until the participants clearly understood the overall activity. Within $30 \mathrm{~s}$, the participants needed to complete each condition consisting of the dominant hand, nondominant hand, and bimanual dexterity. The order of the three conditions of the PPT was randomized by the researchers before testing. Upon completion of PPT, these participants were further explained and demonstrated the assessment of the continuous circle-drawing test. The participants were instructed to draw a circle with two hands simultaneously for a total of three times. Their time spent was recorded in each activity and the average time was then calculated. Afterward, the participants were interviewed face-to-face and instructed to truthfully answer the questions for each item of the GADL questionnaire. The summarized protocol was shown in online supplementary material (see www.karger.com/doi/10.1159/000521644 for all online suppl. material).

\section{Data Analysis}

All data were recorded and entered using the statistical software version 20.0 of SPSS to determine the correlations between hand dexterity and bimanual coordination with each domain of GADL. Descriptive data-included demographic and clinical characteristics were analyzed using percentage and mean \pm SD. A multiple linear regression analysis with a forced stepwise model was used to test whether the performance of hand functions on each hand, bimanual dexterity, and bimanual coordination could predict the score from each domain of GADL including the score of dominant hand dexterity, nondominant hand dexterity, bimanual dexterity, and the elapsed time of bimanual coordination. The association between hand dexterity and bimanual coordination was analyzed using Pearson correlations to evaluate if the contribution
Table 2. Mean values of selected parameters

\begin{tabular}{ll}
\hline Parameters & Mean values \\
\hline Age (years, mean \pm SD) & $68.39 \pm 4.50$ \\
MoCA (score, mean \pm SD) & $20.55 \pm 2.11$ \\
TGDS-15 (score, mean \pm SD) & $2.35 \pm 1.70$ \\
GADL (score, mean \pm SD) & \\
$\quad$ Self-care domain & $10.00 \pm 0.00$ \\
$\quad$ Domestic domain & $7.52 \pm 0.811$ \\
Complex domain & $7.45 \pm 0.675$ \\
PPT (peg, mean \pm SD) & $12.13 \pm 1.59$ \\
$\quad$ Dominant hand & $11.45 \pm 2.14$ \\
$\quad$ Nondominant hand & $6.90 \pm 1.72$ \\
$\quad$ Bimanual hands & $6.40 \pm 2.02$ \\
Bimanual coordination (s, mean \pm SD) & \\
\hline
\end{tabular}

TGDS, Thai geriatric depression scales.

of hand dexterity and bimanual coordination to ADL performance was independent. A value of $p<0.05$ was regarded as statistically significant.

\section{Results}

We evaluated 31 participants who exhibited MCI. The majority of the subjects are of low socioeconomic status, of an age range of 60-69 years old, possess less than 4 years of formal education, and have underlying diseases. The baseline demographic characteristics are shown in Table 1.

Table 2 shows the sociodemographic and clinical data, ADL domain scores, the average score for hand dexterity, and the elapsed time for bimanual coordination. Thirtyone participants who exhibited MCI had an average MoCA score of $20.55 \pm 2.11$. The average age was $68.39 \pm$ 4.50 years; none of them presented depression and anxiety symptoms. The mean TGDS-15 was $2.35 \pm 1.70$.

The PPT scores for the three conditions (dominant hand, nondominant hand, and bimanual dexterity) were $12.13 \pm 1.59,11.45 \pm 2.14$, and $6.90 \pm 1.72$, respectively. The GADL scores for the domestic and complex domains were $7.52 \pm 0.811$ and $7.45 \pm 0.675$, respectively. The average time of bimanual coordination was $6.40 \pm 2.02 \mathrm{~s}$. However, the average score for the self-care domain of the GADL assessment was $10.00 \pm 0.00$, which represented no functional decline in older adults with MCI. Analysis of older adults with MCI should not include the self-care or BADL domain; therefore, we did not include this domain in the data analysis and discussion. 


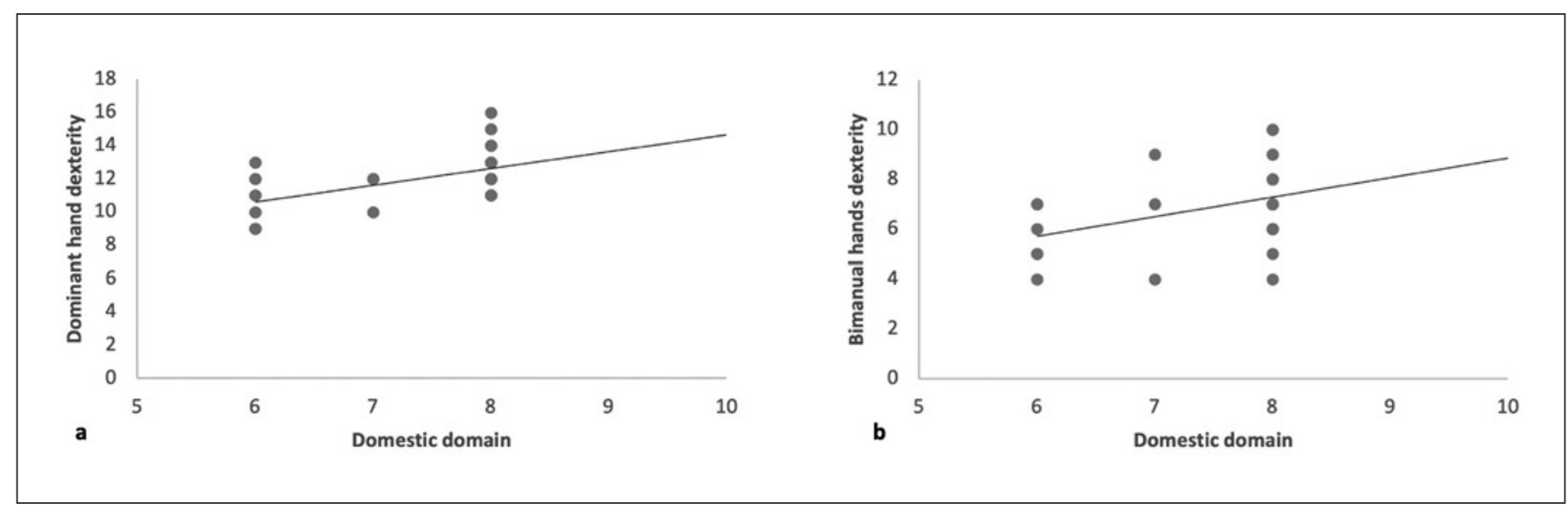

Fig. 2. Association between domestic domain of GADL and dominant hand and bimanual dexterity. The correlations of the domestic domain of GADL showed a significance correlation with the dominant hand (a) and bimanual hands dexterity (b) $(r=0.516,0.372$, respectively). The number of dots in the scatterplot differs from the sample size due to superposed values.

Table 3. Linear regression models of hand dexterity and bimanual coordination as predicted effects of hand performance declination on ADL

\begin{tabular}{|c|c|c|c|c|c|c|}
\hline$F$ & df & $p$ value & $R 2, \%$ & Predictors & Standard ß & $p$ value \\
\hline \multicolumn{7}{|c|}{ GADL: domestic domain } \\
\hline \multirow[t]{4}{*}{10.55} & 30 & 0.003 & 26.7 & Dominant hand dexterity & 0.516 & 0.003 \\
\hline & & & & Nondominant hand dexterity & -0.196 & 0.376 \\
\hline & & & & Bimanual dexterity & 0.209 & 0.230 \\
\hline & & & & Bimanual coordination & 0.025 & 0.881 \\
\hline \multicolumn{7}{|c|}{ GADL: complex domain } \\
\hline \multirow[t]{4}{*}{13.69} & 30 & 0.001 & 32.1 & Dominant hand dexterity & 0.566 & 0.001 \\
\hline & & & & Nondominant hand dexterity & 0.214 & 0.314 \\
\hline & & & & Bimanual dexterity & 0.168 & 0.318 \\
\hline & & & & Bimanual coordination & -0.105 & 0.505 \\
\hline
\end{tabular}

\section{Correlations between Domestic Domain with Hand Dexterity and Coordination}

There was a significant correlation between the domestic domain with dominant hand dexterity $(p=0.001$, $r=0.516)$ and bimanual dexterity $(p=0.020, r=0.372)$ as showed in Figure 2. However, there was no correlation found between nondominant hand dexterity and bimanual coordination with the domestic domain.

In the regression analysis, the model was significant for the domestic domain of GADL $(F=10.55, p=0.003$, $\left.R^{2}=0.267\right)$ and the predictor of the greatest effect for the domestic domain was dominant hand dexterity $(p=$ $0.003)$. Nondominant hand dexterity, bimanual dexterity, and bimanual coordination were not predictors for the domestic domain $(p>0.05)$ as showed in Table 3.

\section{Correlations between Complex Domain with Hand Dexterity and Coordination}

There was a significant correlation between the complex domain with dominant hand dexterity $(p<0.001, r=$ $0.566)$, nondominant hand dexterity $(p=0.002, r=0.500)$, and bimanual dexterity $(p=0.025, r=0.355)$ as shown in Figure 3. However, we did not find any correlation between bimanual coordination with the complex domain.

In the regression analysis, the model was significant for the complex domain of GADL $(F=13.69, p=0.001$, $\left.R^{2}=0.321\right)$ and the predictor of greatest effect for the complex domain was dominant hand dexterity $(p=$ $0.001)$. Nondominant hand dexterity, bimanual dexterity, and bimanual coordination were not predictors for the complex domain $(p>0.05)$ as showed in Table 3. 


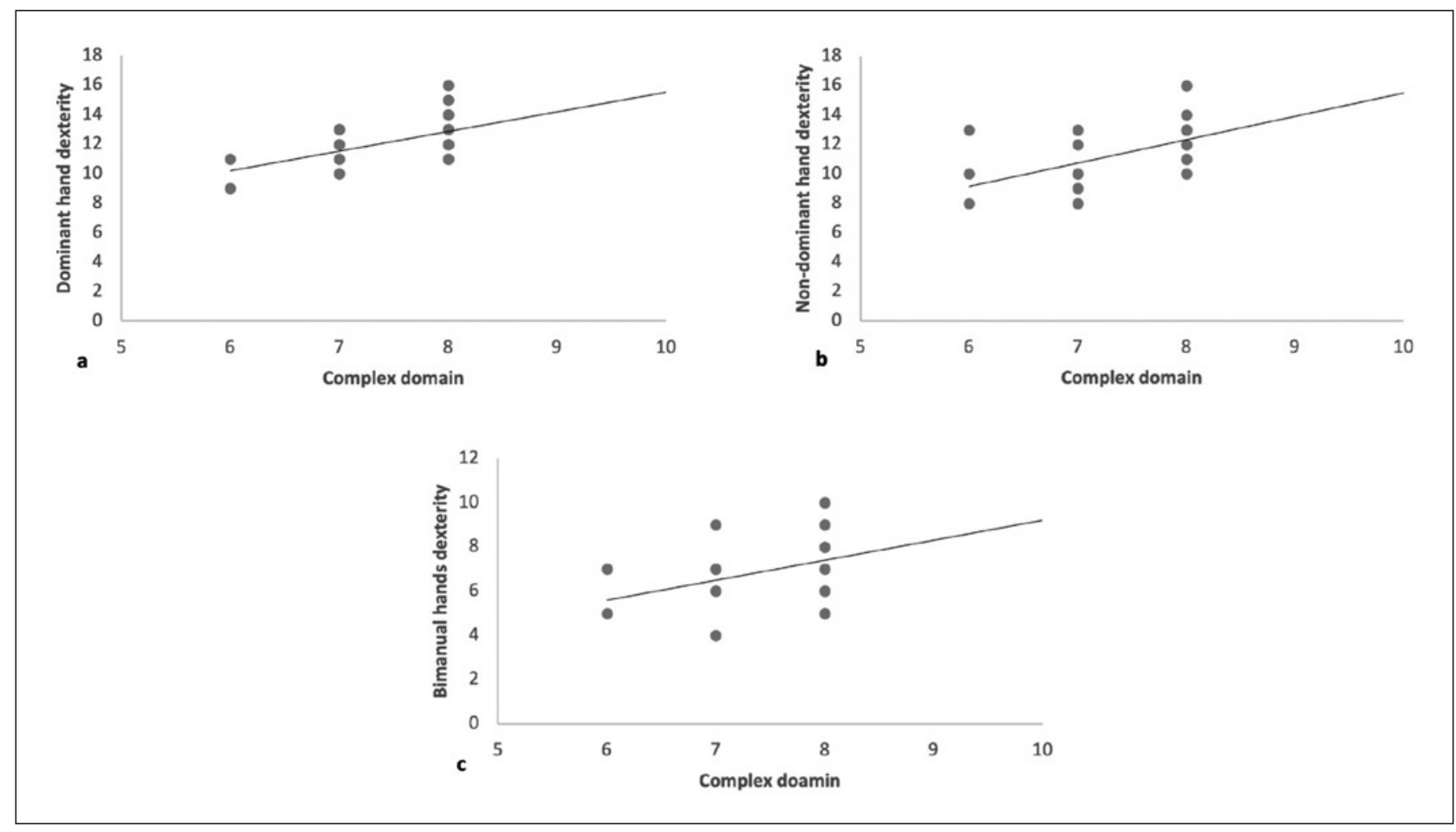

Fig. 3. Association between complex domain of GADL and dominant hand and bimanual dexterity. The correlations of the complex domain of GADL showed a significance correlation with the dominant hand (a), nondominant hand (b), and bimanual hands dexterity $(\mathbf{c})(r=0.566(\mathbf{a}), 0.500(\mathbf{b})$, and 0.355 (c), respectively). The number of dots in the scatterplot differs from the sample size due to superposed values.

\section{Discussion}

The correlation between specific motor declines and poor ADL shows limited reports particularly on older adults with MCI. Our results found correlations between hand dexterity with domestic and complex ADL. Moreover, we found that only dominant hand dexterity had a significant influence on the performance of domestic and complex ADL.

Interestingly, our finding shows a similarity on decreasing the hand dexterity with a previous study that PPT in healthy older adults revealed an asymmetrical reduction of hand dexterity in each and both sides of the right, left and both hands, respectively [30]. Furthermore, the average PPT of the right, left, and both hands was 14.3, 13.7, and 10.9 and $12.7,12.7$, and 10.2 in the age-group of $60-70$ years for females and males, respectively [26]. PPT in the dominant hand in healthy older adults demonstrated the average values of 14.5, 13.7, 13.1, and 11.9 in the age-groups of 60 $64,65-69,70-74$, and $\geq 75$ years, respectively [33].

Effects of Hand Performance Declination on $\mathrm{ADL}$
The asymmetrical declination between hemispheres has been explained in earlier studies. Kalisch et al. [34] designed an experiment to investigate age-related changes in hand dominance in individuals aged 20-90 years. They measured motor performance and recorded the use of the hand in everyday activities. They found that righthand dominance declined with aging and the participants showed more balance between using the right hand and left hand at an advanced age than younger ones [34,35]. A greater decline in hand performance was found in the dominant hand at an advancing age and with older adults who could no longer maintain the intensity and load that they once favored with the dominant hand.

Moreover, similar results also reported the asymmetrical brain declination from a study done by Przybyla et al. [36]. Their findings showed a decreased tendency of the dominant hand and balance with using the right and left hand at an advanced age. Age-related changes in regard to lateral asymmetrical motor reduction occurred in older adults, and dominant hand performance remained the 
same for hand path curvature while nondominant hand performance decreased in older adults compared to younger ones. Moreover, the accuracy and precision of the dominant hand decreased from young adults and showed balance in advanced age [36]. The general finding is that prefrontal cortex activity during cognitive tasks is less lateralized in older adults compared to younger adults, which potentially contributes to the HAROLD model. The greater bilateral recruitment that occurs in older adults was associated with better task performance, whereas those who performed poorly on cognitive tasks exhibited an asymmetrical pattern in their neural activity [36]. It is plausible that the reduced motor asymmetry occurs in older adults. Mattay et al. [37] showed increased ipsilateral activation patterns in older adults when compare to young adults during a simple unilateral pressing task.

The present study found there was no association between bimanual coordination with domestic and complex ADL. This finding is supported by age-related changes in interlimb coordination, as indicated by bilateral motor synergies during isometric force control for two visual conditions (yes and no visual feedback) [38]. It is noteworthy that the older adults revealed higher values of force variability in asymmetry and correlation of interlimb coordination in the older adults than the younger adults [38]. Nondominant hand force variation, controlled by right hemisphere, is responsible for modulating the stability of limb movement. During the bilateral movement, interhemispheric connection by the corpus collosum function balanced interhemispheric inhibition for successful task performance [28]. In addition, a smaller volume of the corpus collosum and reduced white matter integrity was found in older adults [39]. Thus, these structural and functional changes (less interhemispheric connectivity) in the corpus callosum may lead to more functional motor impairments in interlimb coordination [27]. Another possibility was the cerebellum, which controls bilateral motor synergy and enhances motor coordination and online-motor correction based on error learning [40]. Presumably, aging influences cerebellar functions that may directly (or indirectly) interfere with bilateral motor synergies.

Our results suggested that the motor impairment, which concerns hand dexterity or bimanual coordination, was a consequence of cognitive decline in older adults with MCI. Complex ADL concerns tasks that need cognitive function and more stability and precision skills, so it correlates with the nondominant hand to maintain the skills. In particular, the dominant hand dexterity was the most effective predictor for predicting the ability to perform ADL, whether in the domestic or complex domain. However, bimanual coordination did not observe a correlation with ADL. Previous studies have reported that a smaller volume of the corpus collosum may lead to impairment in interlimb coordination in older adults [39].

Our study had limitations that should be addressed. First, the small sample size may not generally provide much significant findings to support the overall study. A large scale of sample size is definitely required in future studies before any conclusion could be made. Second, this was a cross-sectional study that may not be able to complete all information, especially a predictive model on motor function decline. In future studies, we could assess motor declination in long-term motor changes that are affected by ADL or set a comparison group including young adults or healthy older adults to investigate the tendency for changes in motor function. Lastly, we included independent parameters which may not be sufficient to represent all activities in ADL. Therefore, in future studies, we could consider more parameters such as gait speed, balance or eye-hand coordination to verify those associations before any conclusion could be made.

\section{Conclusion}

Based on the results obtained from this study, older adults with MCI showed their abilities on self-care or BADL. Hand dexterity was found in correlation with domestic and complex ADL. Moreover, the dominant hand dexterity strongly affects the domestic and complex domains of ADL. This preliminary study therefore provides important information to offer; practical benefits in using cognitive and motor assessment would allow the clinician to compute better estimate of functional performance and help to predict ADL deterioration in elderly with MCI. Elderly with MCI who impaired motor dexterity may be at higher risk of loss of IADL performance, and physical training program focusing fine motor dexterity may improve performance in these ADLs.

\section{Acknowledgments}

The authors would like to thank to all the participants who sacrificed their time and excellently participated in this study. Moreover, we would especially like to grateful to Miss Piyaphat Chinaphandu for her assistance in data collection. Finally, we would like to give our utmost sincere thanks to the head of senior association in Thasala Sub-distinct, Nakhon Si Thammarat, Thailand.
Rattanawan 


\section{Statement of Ethics}

This study was conducted in conformity with the World Medical Association Declaration of Helsinki and was approved by the Ethics Committee of Walailak University (Reference No. WUEC-20-06901). All participants have agreed with written informed consent.

\section{Author Contributions}

Because this study has an author, Prathomchai Rattanawan designed the study, collected data, planned and performed the statistical analyses, interpreted the results, and wrote the manuscript. The author received suggestions about editing English's grammar from his advisor.

\section{Conflict of Interest Statement}

There is no conflict of interest to declare.

\section{Funding Sources}

This study was supported by Walailak University research Grant (WU-IRG-63-057). The funder had no role in the study design, data collection, discussion, or preparation of the manuscript.

\section{Data Availability Statement}

All data generated or analyzed during this study are included in this Google form link: https://docs.google.com/ spreadsheets/d/1mlUh1nIhZyOaHWj7Q4Er6YvzUwDfxnCZfS6 RArcfJSQ/edit?usp=sharing The link was set to only see.

\section{References}

1 Greiner PA, Snowdon DA, Schmitt FA. The loss of independence in activities of daily living: the role of low normal cognitive function in elderly nuns. Am J Public Health. 1996; 86(1):62-6.

2 McKhann GM, Knopman DS, Chertkow H, Hyman BT, Jack CR Jr, Kawas CH, et al. The diagnosis of dementia due to Alzheimer's disease: recommendations from the National Institute on Aging-Alzheimer's Association workgroups on diagnostic guidelines for Alzheimer's disease. Alzheimers Dement. 2011; 7(3):263-9.

3 Albert MS, DeKosky ST, Dickson D, Dubois B, Feldman HH, Fox NC, et al. The diagnosis of mild cognitive impairment due to $\mathrm{Alz}$ heimer's disease: recommendations from the National Institute on Aging-Alzheimer's Association workgroups on diagnostic guidelines for Alzheimer's disease. Alzheimers Dement. 2011;7(3):270-9.

4 Beauchet O, Allali G, Montero-Odasso M, Sejdić E, Fantino B, Annweiler C. Motor phenotype of decline in cognitive performance among community-dwellers without dementia: population-based study and meta-analysis. PLoS One. 2014;9(6):e99318.

5 Song CS. Relationship between visuo-perceptual function and manual dexterity in community-dwelling older adults. J Phys Ther Sci. 2015;27(6):1871-4.

6 Rodríguez-Aranda C, Mittner M, Vasylenko O. Association between executive functions, working memory, and manual dexterity in young and healthy older adults: an exploratory study. Percept Mot Skills. 2016;122(1): $165-92$.

7 Aggarwal NT, Wilson RS, Beck TL, Bienias JL, Bennett DA. Motor dysfunction in mild cognitive impairment and the risk of incident Alzheimer disease. Arch Neurol. 2006;63(12): 1763-9.
8 de Paula JJ, Albuquerque MR, Lage GM, Bicalho MA, Romano-Silva MA, Malloy-Diniz LF. Impairment of fine motor dexterity in mild cognitive impairment and Alzheimer's disease dementia: association with activities of daily living. Braz J Psychiatry. 2016;38(3): 235-8.

9 Scherder E, Dekker W, Eggermont L. Higherlevel hand motor function in aging and (preclinical) dementia: its relationship with (instrumental) activities of daily life - a mini-review. Gerontology. 2008;54(6):333-41.

10 Yan JH, Rountree S, Massman P, Doody RS, Li H. Alzheimer's disease and mild cognitive impairment deteriorate fine movement control. J Psychiatr Res. 2008;42(14):1203-12.

11 Yan JH, Dick MB. Practice effects on motor control in healthy seniors and patients with mild cognitive impairment and Alzheimer's disease. Neuropsychol Dev Cogn B Aging Neuropsychol Cogn. 2006;13(3-4):385-410.

12 Jekel K, Damian M, Wattmo C, Hausner L, Bullock R, Connelly PJ, et al. Mild cognitive impairment and deficits in instrumental activities of daily living: a systematic review. Alzheimers Res Ther. 2015;7(1):17.

13 Rinne P, Hassan M, Fernandes C, Han E, Hennessy E, Waldman A, et al. Motor dexterity and strength depend upon integrity of the attention-control system. Proc Natl Acad Sci U S A. 2018;115(3):536-45.

14 Marshall GA, Rentz DM, Frey MT, Locascio JJ, Johnson KA, Sperling RA, et al. Executive function and instrumental activities of daily living in mild cognitive impairment and $\mathrm{Alz}$ heimer's disease. Alzheimers Dement. 2011; 7(3):300-8.

15 Greene LS, Williams HG. Aging and coordination from the dynamic pattern perspective. In: Ferrandez AM, Teasdale N, editors. Changes in sensory motor behavior in aging. Advances in psychology; 1996. Vol. 114; p. 89-131.
16 Stelmach GE, Amrhein PC, Goggin NL. Age differences in bimanual coordination. I Gerontol. 1988;43(1):P18-23.

17 Seidler RD, Alberts JL, Stelmach GE. Changes in multi-joint performance with age. Motor Control. 2002;6(1):19-31.

18 Swinnen SP. Intermanual coordination: from behavioural principles to neural-network interactions. Nat Rev Neurosci. 2002;3(5):34859.

19 Gooijers J, Swinnen SP. Interactions between brain structure and behavior: the corpus callosum and bimanual coordination. Neurosci Biobehav Rev. 2014;43:1-19.

20 Pagani E, Agosta F, Rocca MA, Caputo D, Filippi M. Voxel-based analysis derived from fractional anisotropy images of white matter volume changes with aging. Neuroimage. 2008;41(3):657-67.

21 Giorgio A, Santelli L, Tomassini V, Bosnell R, Smith S, De Stefano N, et al. Age-related changes in grey and white matter structure throughout adulthood. Neuroimage. 2010; 51(3):943-51.

22 Frederiksen KS. Corpus callosum in aging and dementia. Dan Med J. 2013;60(10): B4721.

23 Stricker NH, Salat DH, Kuhn TP, Foley JM, Price JS, Westlye LT, et al. Mild cognitive impairment is associated with white matter integrity changes in late-myelinating regions within the corpus callosum. Am J Alzheimers Dis Other Demen. 2016;31(1):68-75.

24 Sullivan EV, Rohlfing T, Pfefferbaum A. Quantitative fiber tracking of lateral and interhemispheric white matter systems in normal aging: relations to timed performance. Neurobiol Aging. 2010;31(3):464-81.

25 Bangert AS, Reuter-Lorenz PA, Walsh CM, Schachter AB, Seidler RD. Bimanual coordination and aging: neurobehavioral implications. Neuropsychologia. 2010;48(4):1165-70. 
26 Shih PC, Steele CJ, Nikulin V, Villringer A, Sehm B. Kinematic profiles suggest differential control processes involved in bilateral inphase and anti-phase movements. Sci Rep. 2019;9:3273.

27 Fujiyama H, Van Soom J, Rens G, Gooijers J, Leunissen I, Levin O, et al. Age-related changes in frontal network structural and functional connectivity in relation to bimanual movement control. J Neurosci. 2016;36(6):180822.

28 Woytowicz E, Whitall J, Westlake KP. Agerelated changes in bilateral upper extremity coordination. Curr Geriatr Rep. 2016;5(3): 191-9.

29 Nasreddine ZS, Phillips NA, Bédirian V, Charbonneau S, Whitehead V, Collin I, et al. The Montreal Cognitive Assessment, MoCA: a brief screening tool for mild cognitive impairment. J Am Geriatr Soc. 2005;53(4):6959.

30 Desrosiers J, Hébert R, Bravo G, Dutil E. The Purdue Pegboard Test: normative data for people aged 60 and over. Disabil Rehabil. 1995;17(5):217-24.
31 Paula JJ, Bertola L, Ávila RT, Assis Lde O, Albuquerque M, Bicalho MA, et al. Development, validity, and reliability of the General Activities of Daily Living Scale: a multidimensional measure of activities of daily living for older people. Braz J Psychiatry. 2014;36(2): 143-52.

32 Rossetti HC, Lacritz LH, Cullum CM, Weiner MF. Normative data for the Montreal Cognitive Assessment (MoCA) in a populationbased sample. Neurology. 2011;77(13):12725.

33 Kobayashi-Cuya KE, Sakurai R, Sakuma N, Suzuki H, Yasunaga M, Ogawa S, et al. Hand dexterity, not handgrip strength, is associated with executive function in Japanese community-dwelling older adults: a cross-sectional study. BMC Geriatr. 2018;18(1):192.

34 Kalisch T, Wilimzig C, Kleibel N, Tegenthoff M, Dinse HR. Age-related attenuation of dominant hand superiority. PLoS One. 2006; 1(1):e90.
35 Sebastjan A, Skrzek A, Ignasiak Z, Sławińska T. Age-related changes in hand dominance and functional asymmetry in older adults. PLoS One. 2017;12(5):e0177845.

36 Przybyla A, Haaland KY, Bagesteiro LB, Sainburg RL. Motor asymmetry reduction in older adults. Neurosci Lett. 2011;489(2):99-104.

37 Mattay VS, Fera F, Tessitore A, Hariri AR, Das S, Callicott JH, et al. Neurophysiological correlates of age-related changes in human motor function. Neurology. 2002;58(4):6305.

38 Kang N, Roberts LM, Aziz C, Cauraugh JH. Age-related deficits in bilateral motor synergies and force coordination. BMC Geriatr. 2019; 19:287.

39 Fling BW, Walsh CM, Bangert AS, ReuterLorenz PA, Welsh RC, Seidler RD. Differential callosal contributions to bimanual control in young and older adults. J Cogn Neurosci. 2011;23(9):2171-85.

40 Seidler RD, Kwak Y, Fling BW, Bernard JA. Neurocognitive mechanisms of error-based motor learning. Adv Exp Med Biol. 2013;782: 39-60. 